PROCEEDINGS OF THE

AMERICAN MATHEMATICAL SOCIETY

Volume 137, Number 2, February 2009, Pages 685-694

S 0002-9939(08)09670-6

Article electronically published on September 16, 2008

\title{
REMARKS ON THE VANISHING OBSTACLE LIMIT FOR A 3D VISCOUS INCOMPRESSIBLE FLUID
}

\author{
DRAGOŞ IFTIMIE AND JAMES P. KELLIHER
}

(Communicated by Walter Craig)

\begin{abstract}
In [Math. Ann. 336 (2006), 449-489], the authors consider the two-dimensional Navier-Stokes equations in the exterior of an obstacle shrinking to a point and determine the limit velocity. Here we consider the same problem in the three-dimensional case, proving that the limit velocity is a solution of the Navier-Stokes equations in the full space.
\end{abstract}

\section{INTRODUCTION}

The investigation of small obstacle limits in an incompressible fluid was initiated in [3. In that paper, the authors consider the Euler equations in the exterior of a two-dimensional obstacle that shrinks homothetically (that is, by dilation) to a point. It is assumed that the initial vorticity is the restriction to the exterior of the obstacle of a smooth vorticity field compactly supported in $\mathbb{R}^{2} \backslash\{0\}$, and that the circulation of the velocity on the boundary of the obstacle is independent of the size of the obstacle. It is then proved in [3] that the limit velocity is a solution of a PDE that looks like an Euler equation that embeds the Dirac mass of the point the obstacle shrinks to. The vorticity also acquires a Dirac mass at this point. The case of several obstacles was treated in [5] and the two-dimensional viscous case in 4, where it is proved that the limit equation is also Navier-Stokes but there is still formation of an additional Dirac mass in the limit vorticity. This is due to the circulation of the velocity on the boundary of the obstacle not vanishing.

Here we consider the same problem in the three-dimensional case: pass to the limit in the Navier-Stokes equations in the exterior of an obstacle that shrinks to a point. In contrast to the two-dimensional case, we do not have to prescribe the circulation of the velocity on the boundary since the domain is simply connected. We prove that the limit equation is the Navier-Stokes equation in the full space and that the vorticity of the limit velocity at time $t=0$ is simply the initial vorticity that we give for the obstacle-dependent problem. Therefore, there is no formation of additional vorticity as in the case of $\mathbb{R}^{2}$. We are also able to consider more general obstacles than in [4]: instead of assuming that the obstacle homothetically shrinks to a point, we assume only that the diameter of the obstacle goes to zero.

Received by the editors January 18, 2008.

2000 Mathematics Subject Classification. Primary 76D05.

Key words and phrases. Navier-Stokes equations.

The second author was supported in part by NSF grant DMS-0705586 during the period of this work.

(C)2008 American Mathematical Society Reverts to public domain 28 years from publication 
More precisely, let $\Pi_{\varepsilon}=\mathbb{R}^{3} \backslash \bar{\Omega}_{\varepsilon}$ be a simply connected exterior domain with $C^{\infty}$ boundary such that $\bar{\Omega}_{\varepsilon} \subset B(0, M \varepsilon)$, where the constant $M$ is independent of $\varepsilon$. We assume that the initial vorticity $\omega_{0}$ is smooth, divergence-free, and compactly supported in $\mathbb{R}^{3}$. Since the domain $\Pi_{\varepsilon}$ is simply connected, there exists a unique square-integrable, divergence-free velocity field $u_{0}^{\varepsilon}$ in $\Pi_{\varepsilon}$ tangent to the boundary of $\Pi_{\varepsilon}$ and whose curl is $\left.\omega_{0}\right|_{\Pi_{\varepsilon}}$ (see Proposition 6 below). We also denote by $u_{0}$ the velocity defined on $\mathbb{R}^{3}$ which is associated to the vorticity $\omega_{0}$ :

$$
u_{0}(x)=-\int_{\mathbb{R}^{3}} \frac{x-y}{4 \pi|x-y|^{3}} \times \omega_{0}(y) d y,
$$

where $\times$ denotes the standard cross product of vectors in $\mathbb{R}^{3}$.

Let $u^{\varepsilon}=u^{\varepsilon}(t, x)$ be a weak Leray solution of the Navier-Stokes equations in $\Pi_{\varepsilon}$ with initial velocity $u_{0}^{\varepsilon}$ and homogeneous Dirichlet boundary conditions:

$$
\begin{cases}\partial_{t} u^{\varepsilon}-\nu \Delta u^{\varepsilon}+u^{\varepsilon} \cdot \nabla u^{\varepsilon}=-\nabla p^{\varepsilon} & \text { in } \Pi_{\varepsilon} \times(0, \infty), \\ \operatorname{div} u^{\varepsilon}=0 & \text { in } \Pi_{\varepsilon} \times[0, \infty), \\ u^{\varepsilon}=0 & \text { on } \partial \Pi_{\varepsilon} \times(0, \infty), \\ u^{\varepsilon}(0, \cdot)=u_{0}^{\varepsilon} & \text { in } \Pi_{\varepsilon} .\end{cases}
$$

Such a weak solution is well-known to exist; see for example [2]. The aim of this paper is to prove the following theorem.

Theorem 1. Let $u_{0}, \omega_{0}$, and $u_{0}^{\varepsilon}$ be as defined above. Let $u^{\varepsilon}$ be a weak Leray solution of the Navier-Stokes equations on $\Pi_{\varepsilon}$ with initial velocity $u_{0}^{\varepsilon}$ and denote by $\widetilde{u}^{\varepsilon}$ the extension of $u^{\varepsilon}$ to $\mathbb{R}^{3}$ with value 0 on $\bar{\Omega}_{\varepsilon}$. There exists a subsequence of $\widetilde{u}^{\varepsilon}$ that converges strongly in $L_{\text {loc }}^{2}\left([0, \infty) \times \mathbb{R}^{3}\right)$ to a weak Leray solution of the Navier-Stokes equations in $\mathbb{R}^{3}$ with initial velocity $u_{0}$.

The proof of this result consists of two parts. We prove first that $u_{0}^{\varepsilon}$ converges to $u_{0}$ strongly in $L^{2}$; see Proposition 6 below. We then conclude by showing in Theorem 7 that strong convergence in $L^{2}$ for the initial velocity implies convergence of solutions in the vanishing obstacle limit.

The regularity of the initial vorticity can be lowered considerably and still obtain convergence as in Theorem 1 . We briefly discuss this in Section 4.

\section{Notation AND PRELIMINARY RESUlts}

For a function $f$ defined on $\Pi_{\varepsilon}$, we denote by $\tilde{f}$ the function defined on $\mathbb{R}^{3}$ which vanishes on $\bar{\Omega}_{\varepsilon}$ and equals $f$ on $\Pi_{\varepsilon}$. If $f$ is regular enough and vanishes on $\partial \Omega_{\varepsilon}$, then one has that $\nabla \widetilde{f}=\widetilde{\nabla f}$ in $\mathbb{R}^{3}$. If $v$ is a regular enough vector field defined on $\Pi_{\varepsilon}$ and tangent to $\partial \Omega_{\varepsilon}$, then one has that $\operatorname{div} \widetilde{v}=\widetilde{\operatorname{div} v}$ in $\mathbb{R}^{3}$. In particular, we have that $\operatorname{div} \widetilde{u}_{0}^{\varepsilon}=0$ in $\mathbb{R}^{3}$. We denote by $H_{\varepsilon}$ the space of square-integrable vector fields on $\Pi_{\varepsilon}$ which are divergence-free and tangent to the boundary. We will also use the classical Sobolev space $H^{m}$ and the space $C_{b}^{m}$ of bounded functions having bounded derivates up to the order $m$.

Definition 2. We say that $u^{\varepsilon}$ is a weak Leray solution of (2) if

$$
\left.u^{\varepsilon} \in C_{w}^{0}\left([0, \infty) ; H_{\varepsilon}\right)\right) \cap L^{\infty}\left([0, \infty) ; H_{\varepsilon}\right) \cap L_{l o c}^{2}\left([0, \infty) ; H_{0}^{1}\left(\Pi_{\varepsilon}\right)\right)
$$

verifies the equation in the sense of distributions, i.e.

$$
(3)-\int_{0}^{\infty} \int_{\Pi_{\varepsilon}} u^{\varepsilon} \cdot \partial_{t} \varphi+\nu \int_{0}^{\infty} \int_{\Pi_{\varepsilon}} \nabla u^{\varepsilon} \cdot \nabla \varphi+\int_{0}^{\infty} \int_{\Pi_{\varepsilon}} u^{\varepsilon} \cdot \nabla u^{\varepsilon} \cdot \varphi=\int_{\Pi_{\varepsilon}} u_{0}^{\varepsilon} \cdot \varphi(0)
$$


for every divergence-free vector field $\varphi \in C_{0}^{\infty}\left([0, \infty) \times \Pi_{\varepsilon}\right)$, and moreover $u^{\varepsilon}$ satisfies the following energy inequality:

$$
\left\|u^{\varepsilon}(t)\right\|_{L^{2}\left(\Pi_{\varepsilon}\right)}^{2}+2 \nu \int_{0}^{t}\left\|\nabla u^{\varepsilon}\right\|_{L^{2}\left(\Pi_{\varepsilon}\right)}^{2} \leq\left\|u_{0}^{\varepsilon}\right\|_{L^{2}\left(\Pi_{\varepsilon}\right)}^{2} \quad \forall t \geq 0 .
$$

Above $C_{w}^{0}$ denotes the space of weakly continuous functions. We will use a similar definition for weak Leray solutions on $\mathbb{R}^{3}$.

For a vector field $\varphi$ in $\mathbb{R}^{3}$ we define a stream function $\psi=T \varphi$ by

$$
\psi(x)=(T \varphi)(x)=(S \varphi)(x)-(S \varphi)(0), \quad(S \varphi)(x)=-\int_{\mathbb{R}^{3}} \frac{x-y}{4 \pi|x-y|^{3}} \times \varphi(y) d y .
$$

We will use the following properties of the operator $T$.

Lemma 3. Suppose that $\varphi$ is a vector field belonging to $L^{2}\left(\mathbb{R}^{3}\right) \cap L^{4}\left(\mathbb{R}^{3}\right)$. Then $T \varphi \in C_{b}^{0}\left(\mathbb{R}^{3}\right)$ and $\|T \varphi\|_{L^{\infty}\left(\mathbb{R}^{3}\right)} \leq C\|\varphi\|_{L^{2} \cap L^{4}}$. Moreover, if $\operatorname{div} \varphi=0$, then $\operatorname{curl} T \varphi=$ $\varphi$. Finally, for all $m \geq 1$, the operator $T$ is bounded from $H^{m}\left(\mathbb{R}^{3}\right)$ into $C_{b}^{m-1}\left(\mathbb{R}^{3}\right)$.

Proof. We decompose

$(S \varphi)(x)=-\int_{\mathbb{R}^{3}} \frac{x-y}{4 \pi|x-y|^{3}} \times \varphi(y) d y=\int_{|x-y| \leq 1} \cdots+\int_{|x-y|>1} \cdots \equiv I_{1}(x)+I_{2}(x)$.

One has that $\frac{x}{|x|^{3}} \chi_{|x| \leq 1} \in L^{4 / 3}\left(\mathbb{R}^{3}\right)$ and $\frac{x}{|x|^{3}} \chi_{|x|>1} \in L^{2}\left(\mathbb{R}^{3}\right)$, so we obtain from Young's inequality that

$$
\left\|I_{1}\right\|_{L^{\infty}\left(\mathbb{R}^{3}\right)} \leq C\left\|\frac{x}{|x|^{3}} \chi_{|x| \leq 1}\right\|_{L^{4 / 3}\left(\mathbb{R}^{3}\right)}\|\varphi\|_{L^{4}\left(\mathbb{R}^{3}\right)} \leq C\|\varphi\|_{L^{4}\left(\mathbb{R}^{3}\right)}
$$

and

$$
\left\|I_{2}\right\|_{L^{\infty}\left(\mathbb{R}^{3}\right)} \leq C\left\|\frac{x}{|x|^{3}} \chi_{|x|>1}\right\|_{L^{2}\left(\mathbb{R}^{3}\right)}\|\varphi\|_{L^{2}\left(\mathbb{R}^{3}\right)} \leq C\|\varphi\|_{L^{2}\left(\mathbb{R}^{3}\right)}
$$

together with the continuity of $I_{1}$ and $I_{2}$. Since $\|T \varphi\|_{L^{\infty}\left(\mathbb{R}^{3}\right)} \leq 2\|S \varphi\|_{L^{\infty}\left(\mathbb{R}^{3}\right)}$ we obtain the desired continuity and uniform bound for $T \varphi$.

Suppose now that $\varphi \in H^{m}\left(\mathbb{R}^{3}\right)$. For any multiindex $\alpha$ of order $0<|\alpha| \leq m-1$ we have $\partial^{\alpha} T \varphi=S \partial^{\alpha} \varphi$ and $\partial^{\alpha} \varphi \in H^{1}\left(\mathbb{R}^{3}\right) \hookrightarrow L^{2} \cap L^{4}$. From the first part of the proof, we deduce that $S \partial^{\alpha} \varphi \in C_{b}^{0}$, that is, $T \varphi \in C^{m-1}\left(\mathbb{R}^{3}\right)$ together with the desired bound.

Finally, assume that $\varphi$ is divergence-free. If $\varphi$ is compactly supported, then clearly $S \varphi=\operatorname{curl} F$, where $F=\frac{1}{4 \pi|x|} * \varphi$. One has that $\operatorname{div} F=\frac{1}{4 \pi|x|} * \operatorname{div} \varphi=0$. Then

$$
\operatorname{curl} T \varphi=\operatorname{curl} S \varphi=\operatorname{curl} \operatorname{curl} F=-\triangle F+\nabla \operatorname{div} F=-\triangle\left(\frac{1}{4 \pi|x|}\right) * \varphi=\delta * \varphi=\varphi .
$$

If $\varphi$ is not compactly supported, then there exists a sequence of divergence-free compactly supported vector fields $\varphi_{n} \rightarrow \varphi$ in $L^{2} \cap L^{4}$. Passing to the limit $n \rightarrow \infty$ in $\operatorname{curl} T \varphi_{n}=\varphi_{n}$ implies that $\operatorname{curl} T \varphi=\varphi$. This completes the proof of Lemma 3 .

We will use in Section 3 the following approximation of smooth compactly supported divergence-free vector fields. Let $\varphi$ be a vector field and $\eta \in C^{\infty}\left(\mathbb{R}^{3}\right)$ be such that $\eta \equiv 0$ on $B(0, M)$ and $\eta \equiv 1$ on $\mathbb{R}^{3} \backslash B(0,2 M)$. We define $\eta_{\varepsilon}(x)=\eta(x / \varepsilon)$ and $\varphi_{\varepsilon}=\operatorname{curl}\left(\eta_{\varepsilon} \psi\right)$ with $\psi=T \varphi$ as before. We collect in the following lemma several properties relating $\varphi_{\varepsilon}$ to $\varphi$.

Lemma 4. Let $\varphi \in C_{0}^{\infty}\left(\mathbb{R}^{3}\right)$ be a divergence-free vector field and define $\varphi_{\varepsilon}$ as above. Then 
(i) $\varphi_{\varepsilon}$ is smooth, compactly supported, divergence-free, and vanishes in a neighborhood of the obstacle $\bar{\Omega}_{\varepsilon}$;

(ii) $\varphi_{\varepsilon} \rightarrow \varphi$ strongly in $H^{1}\left(\mathbb{R}^{3}\right)$;

(iii) there exists a constant $C$ independent of $\varepsilon$ such that $\left\|\varphi_{\varepsilon}\right\|_{L^{\infty}\left(\mathbb{R}^{3}\right)}+$ $\left\|\varphi_{\varepsilon}\right\|_{H^{1}\left(\mathbb{R}^{3}\right)} \leq C\|\varphi\|_{H^{3}\left(\mathbb{R}^{3}\right)} ;$

(iv) one can decompose $\nabla \varphi_{\varepsilon}=\xi_{\varepsilon}+\Xi_{\varepsilon}$ with $\xi_{\varepsilon} \rightarrow \nabla \varphi$ weak* in $L^{\infty}\left(\mathbb{R}^{3}\right)$ and $\Xi_{\varepsilon} \rightarrow 0$ strongly in $L^{2}\left(\mathbb{R}^{3}\right)$ and there exists a compact set $L$ independent of $\varepsilon$ such that $\operatorname{supp} \xi_{\varepsilon}, \operatorname{supp} \Xi_{\varepsilon} \subset L$ for all $\varepsilon \leq 1$.

Remark 5. It will be clear from the proof below that we can allow a time dependence in $\varphi$. The results of this lemma hold true uniformly with respect to the time variable.

Proof. We will repeatedly use in this proof that $\psi(0)=0$, so

$$
\|\psi\|_{L^{\infty}(B(0,2 \varepsilon M))} \leq 2 \varepsilon M\|\nabla \psi\|_{L^{\infty}\left(\mathbb{R}^{3}\right)} \leq C \varepsilon\|\varphi\|_{H^{2}} .
$$

Part (ii) follows immediately from the localization properties of $\eta_{\varepsilon}$ and from Lemma 3

To prove (iil) we observe from the explicit expression for $\eta_{\varepsilon}$ that $\eta_{\varepsilon}-1$ and $\nabla \eta_{\varepsilon}$ converge to 0 in $L^{2}$ and $\left\|\nabla^{2} \eta_{\varepsilon}\right\|_{L^{2}\left(\mathbb{R}^{3}\right)}=\varepsilon^{-\frac{1}{2}}\left\|\nabla^{2} \eta\right\|_{L^{2}\left(\mathbb{R}^{3}\right)}$. Since $\varphi_{\varepsilon}=\eta_{\varepsilon} \varphi+\nabla \eta_{\varepsilon} \times \psi$ we have that

$$
\begin{aligned}
\left\|\varphi_{\varepsilon}-\varphi\right\|_{L^{2}\left(\mathbb{R}^{3}\right)} \leq & \left\|\left(\eta_{\varepsilon}-1\right) \varphi\right\|_{L^{2}\left(\mathbb{R}^{3}\right)}+\left\|\nabla \eta_{\varepsilon} \times \psi\right\|_{L^{2}\left(\mathbb{R}^{3}\right)} \\
& \leq\left\|\eta_{\varepsilon}-1\right\|_{L^{2}\left(\mathbb{R}^{3}\right)}\|\varphi\|_{L^{\infty}\left(\mathbb{R}^{3}\right)}+\left\|\nabla \eta_{\varepsilon}\right\|_{L^{2}\left(\mathbb{R}^{3}\right)}\|\psi\|_{L^{\infty}\left(\mathbb{R}^{3}\right)} \stackrel{\varepsilon \rightarrow 0}{\longrightarrow} 0,
\end{aligned}
$$

and similarly,

$$
\begin{aligned}
& \left\|\nabla\left(\varphi_{\varepsilon}-\varphi\right)\right\|_{L^{2}\left(\mathbb{R}^{3}\right)} \leq\left\|\eta_{\varepsilon}-1\right\|_{L^{2}\left(\mathbb{R}^{3}\right)}\|\nabla \varphi\|_{L^{\infty}\left(\mathbb{R}^{3}\right)} \\
& +C\left\|\nabla \eta_{\varepsilon}\right\|_{L^{2}\left(\mathbb{R}^{3}\right)}\left(\|\varphi\|_{L^{\infty}\left(\mathbb{R}^{3}\right)}+\|\nabla \psi\|_{L^{\infty}\left(\mathbb{R}^{3}\right)}\right) \\
& +C\left\|\nabla^{2} \eta_{\varepsilon}\right\|_{L^{2}\left(\mathbb{R}^{3}\right)}\|\psi\|_{L^{\infty}(B(0,2 \varepsilon M))} \stackrel{\varepsilon \rightarrow 0}{\longrightarrow} 0 .
\end{aligned}
$$

The $H^{1}$ bound in part (iii) follows from the estimates above while the uniform bound is an immediate consequence of (5) and of the decomposition $\varphi_{\varepsilon}=\eta_{\varepsilon} \varphi+$ $\nabla \eta_{\varepsilon} \times \psi$.

To prove (iv) we set $\xi_{\varepsilon}=\eta_{\varepsilon} \nabla \varphi$ and $\Xi_{\varepsilon}=\nabla \varphi_{\varepsilon}-\eta_{\varepsilon} \nabla \varphi$ so that $\operatorname{supp} \xi_{\varepsilon} \subset \operatorname{supp} \varphi$. The term $\Xi_{\varepsilon}$ is similar to the expressions estimated above, so it can be proved in the same way that it converges to 0 in $L^{2}$ as $\varepsilon \rightarrow 0$. The sequence $\xi_{\varepsilon}$ is bounded in $L^{\infty}$ and converges to $\nabla \varphi$ in $L^{2}$. Since $\xi_{\varepsilon} \rightarrow \nabla \varphi$ in $L^{2}$ we deduce first that $\int\left(\xi_{\varepsilon}-\nabla \varphi\right) \cdot h \rightarrow 0$ for all $h \in C_{0}^{\infty}\left(\mathbb{R}^{3}\right)^{9}$. Since $\xi_{\varepsilon}$ is bounded in $L^{\infty}$ and $C_{0}^{\infty}$ is dense in $L^{1}$ we infer that $\int\left(\xi_{\varepsilon}-\nabla \varphi\right) \cdot h \rightarrow 0$ for all $h \in L^{1}\left(\mathbb{R}^{3}\right)^{9}$, i.e. that $\xi_{\varepsilon} \rightarrow \nabla \varphi$ weak* in $L^{\infty}$. Finally, the condition on the supports is trivially verified by construction. This completes the proof of the lemma.

We prove next a convergence result for the initial velocities.

Proposition 6. Let $\omega_{0}$ be a divergence-free vector field in $C_{0}^{\infty}\left(\mathbb{R}^{3}\right)$ with $u_{0}$ given by Equation (11). For all $\varepsilon>0$ there exists exactly one vector field $u_{0}^{\varepsilon} \in H_{\varepsilon}$ such that curl $u_{0}^{\varepsilon}=\left.\omega_{0}\right|_{\Pi_{\varepsilon}}$ and there exists a constant $C$ independent of $\varepsilon$ such that $\left\|\widetilde{u}_{0}^{\varepsilon}-u_{0}\right\|_{L^{2}\left(\mathbb{R}^{3}\right)} \leq C \varepsilon^{\frac{3}{2}}$. 
Proof. By the Leray-Helmholtz-Weyl decomposition there exist $u_{0}^{\varepsilon}$ in $H_{\varepsilon}$ and $p$ in $\dot{H}^{1}\left(\Pi_{\varepsilon}\right)=\left\{p \in L_{l o c}^{2}\left(\Pi_{\varepsilon}\right) ; \nabla p \in L^{2}\left(\Pi_{\varepsilon}\right)\right\}$ such that $\left.u_{0}\right|_{\Pi_{\varepsilon}}=u_{0}^{\varepsilon}+\nabla p$ on $\Pi_{\varepsilon}$ with $u_{0}^{\varepsilon}$ and $\nabla p$ unique in these spaces (see, for instance, Theorem 1.1 on p. 107 of [1]). Since the curl of a gradient is zero, $\operatorname{curl} u_{0}^{\varepsilon}=\left.\operatorname{curl} u_{0}\right|_{\Pi_{\varepsilon}}=\left.\omega_{0}\right|_{\Pi_{\varepsilon}}$ in $\Pi_{\varepsilon}$.

Now let $w$ be any vector field in $H_{\varepsilon}$ with $\operatorname{curl} w=\left.\omega_{0}\right|_{\Pi_{\varepsilon}}$ in $\Pi_{\varepsilon}$. Then $w-u_{0}^{\varepsilon}$ is in $H_{\varepsilon}$ with $\operatorname{curl}\left(w-u_{0}^{\varepsilon}\right)=0$, so since $\Pi_{\varepsilon}$ is simply connected, $w-u_{0}^{\varepsilon}=\nabla q$ for some $q$ in $\dot{H}^{1}\left(\Pi_{\varepsilon}\right)$. Also, $\Delta q=\operatorname{div} w-\operatorname{div} u_{0}^{\varepsilon}=0$ on $\Pi_{\varepsilon}$ and $\nabla q \cdot \mathbf{n}=w \cdot \mathbf{n}=0$ on $\partial \Pi_{\varepsilon}$, where $\mathbf{n}$ is the outward unit normal to the boundary. By the uniqueness of the solution to the Neumann problem, $q$ is a constant, so $\nabla q=0$ and $u_{0}^{\varepsilon}=w$, giving the uniqueness of $u_{0}^{\varepsilon}$.

Noting that $u_{0}^{\varepsilon}$ is the $L^{2}$-orthogonal projection of $\left.u_{0}\right|_{\Pi_{\varepsilon}}$ on $H_{\varepsilon}$, we have

$$
\left\|u_{0}^{\varepsilon}-\left.u_{0}\right|_{\Pi_{\varepsilon}}\right\|_{L^{2}\left(\Pi_{\varepsilon}\right)} \leq\left\|w_{\varepsilon}-\left.u_{0}\right|_{\Pi_{\varepsilon}}\right\|_{L^{2}\left(\Pi_{\varepsilon}\right)} \text { for all } w_{\varepsilon} \in H_{\varepsilon} .
$$

Making the particular choice,

$$
w_{\varepsilon}=\operatorname{curl}\left(\eta_{\varepsilon} \psi\right)=\eta_{\varepsilon} u_{0}+\nabla \eta_{\varepsilon} \times \psi, \quad \psi=T u_{0},
$$

we see that $w_{\varepsilon}$ vanishes on $\partial \prod_{\varepsilon}$ and since $w_{\varepsilon}$ is a curl it is also divergence-free. Equation (6) with Lemma 3 then yields

$$
\begin{aligned}
\left\|\widetilde{u}_{0}^{\varepsilon}-u_{0}\right\|_{L^{2}\left(\mathbb{R}^{3}\right)} & \leq\left\|u_{0}^{\varepsilon}-\left.u_{0}\right|_{\Pi_{\varepsilon}}\right\|_{L^{2}\left(\Pi_{\varepsilon}\right)}+\left\|u_{0}\right\|_{L^{2}\left(\Omega_{\varepsilon}\right)} \\
& \leq\left\|\nabla \eta_{\varepsilon} \times \psi\right\|_{L^{2}\left(\Pi_{\varepsilon}\right)}+\left\|\left(1-\eta_{\varepsilon}\right) u_{0}\right\|_{L^{2}\left(\Pi_{\varepsilon}\right)}+\left\|u_{0}\right\|_{L^{2}\left(\Omega_{\varepsilon}\right)} \\
& \leq\left\|\nabla \eta_{\varepsilon}\right\|_{L^{2}}\|\psi\|_{L^{\infty}(B(0,2 M \varepsilon))}+2\left\|u_{0}\right\|_{L^{2}(B(0,2 M \varepsilon))} \\
& \leq C \varepsilon^{3 / 2}\|\nabla \psi\|_{L^{\infty}\left(\mathbb{R}^{3}\right)}+C \varepsilon^{3 / 2}\left\|u_{0}\right\|_{L^{\infty}\left(\mathbb{R}^{3}\right)} \\
& \leq C \varepsilon^{3 / 2}\left\|\omega_{0}\right\|_{H^{3}\left(\mathbb{R}^{3}\right)} .
\end{aligned}
$$

We used above that $\psi(0)=0$.

\section{Proof of the convergence of solutions}

The aim of this section is to prove a general convergence result: strong convergence in $L^{2}$ for the initial data implies convergence of weak Leray solutions in the vanishing obstacle limit. Such a convergence result is classical on a fixed domain; the difficulty here is to deal with the singularity induced by the obstacle that shrinks to a point. Throughout this section we drop the previous assumptions on the initial vorticity and the special forms of the initial velocities $u_{0}^{\varepsilon}$ and $u_{0}$. We will prove the following independent result.

Theorem 7. Suppose that $u_{0}^{\varepsilon} \in H_{\varepsilon}$ and that $u_{0} \in L^{2}\left(\mathbb{R}^{3}\right)$ is a divergence-free vector field such that $\widetilde{u}_{0}^{\varepsilon} \rightarrow u_{0}$ strongly in $L^{2}\left(\mathbb{R}^{3}\right)$. Let $u^{\varepsilon}$ be a weak Leray solution of the Navier-Stokes equations on $\Pi_{\varepsilon}$ with initial velocity $u_{0}^{\varepsilon}$. There exists a subsequence of $\widetilde{u}^{\varepsilon}$ that converges strongly in $L_{l o c}^{2}\left([0, \infty) \times \mathbb{R}^{3}\right)$ to a weak Leray solution of the Navier-Stokes equations in $\mathbb{R}^{3}$ with initial velocity $u_{0}$.

We proceed now with the proof of this theorem. We will use the notation introduced in Section 2, Since $u^{\varepsilon}$ is a weak Leray solution and $u_{0}^{\varepsilon}$ is bounded in $L^{2}\left(\Pi_{\varepsilon}\right)$, the energy inequality (44) implies that $u^{\varepsilon}$ is bounded in $L^{\infty}\left(\mathbb{R}_{+} ; L^{2}\left(\Pi_{\varepsilon}\right)\right) \cap$ $L_{l o c}^{2}\left([0, \infty) ; H^{1}\left(\Pi_{\varepsilon}\right)\right)$. We require now some temporal estimates for $u^{\varepsilon}$. 
3.1. Temporal estimates. Let $\phi \in C_{0}^{\infty}\left(\mathbb{R}^{3}\right)$ be a divergence-free vector field. We construct $\phi_{\varepsilon}$ as in Section 2] It follows from Definition 2, using a standard approximation argument for the test function $\phi_{\varepsilon}$ and taking advantage of the weak continuity in time of $u$ in $L^{2}\left(\Pi_{\varepsilon}\right)$, that

$$
\begin{aligned}
& \left|\left\langle u^{\varepsilon}(t), \phi_{\varepsilon}\right\rangle-\left\langle u^{\varepsilon}(s), \phi_{\varepsilon}\right\rangle\right|=\left|\nu \int_{s}^{t} \int_{\Pi_{\varepsilon}} \nabla u^{\varepsilon} \cdot \nabla \phi_{\varepsilon}+\int_{s}^{t} \int_{\Pi_{\varepsilon}} u^{\varepsilon} \cdot \nabla u^{\varepsilon} \cdot \phi_{\varepsilon}\right| \\
& \quad \leq \nu \int_{s}^{t}\left\|\nabla u^{\varepsilon}\right\|_{L^{2}\left(\Pi_{\varepsilon}\right)}\left\|\nabla \phi_{\varepsilon}\right\|_{L^{2}\left(\mathbb{R}^{3}\right)}+\int_{s}^{t}\left\|u^{\varepsilon}\right\|_{L^{2}\left(\Pi_{\varepsilon}\right)}\left\|\nabla u^{\varepsilon}\right\|_{L^{2}\left(\Pi_{\varepsilon}\right)}\left\|\phi_{\varepsilon}\right\|_{L^{\infty}\left(\mathbb{R}^{3}\right)} \\
& \quad \leq C(t-s)^{\frac{1}{2}}\|\phi\|_{H^{3}\left(\mathbb{R}^{3}\right)}\left\|\nabla u^{\varepsilon}\right\|_{L^{2}\left(\mathbb{R}_{+} \times \Pi_{\varepsilon}\right)}\left(1+\left\|u^{\varepsilon}\right\|_{L^{\infty}\left(\mathbb{R}_{+} ; L^{2}\left(\Pi_{\varepsilon}\right)\right)}\right) \\
& \quad \leq C(t-s)^{\frac{1}{2}}\|\phi\|_{H^{3}\left(\mathbb{R}^{3}\right)}\left\|u_{0}^{\varepsilon}\right\|_{L^{2}\left(\Pi_{\varepsilon}\right)}\left(1+\left\|u_{0}^{\varepsilon}\right\|_{L^{2}\left(\Pi_{\varepsilon}\right)}\right),
\end{aligned}
$$

where we used (4), and Lemma 4 (iii), where the constant $C$ is independent of $\varepsilon, s$ and $t$ (though it depends on $\nu$ ). For $t \in \mathbb{R}_{+}$let us define $F_{\varepsilon}(t) \in \mathcal{D}^{\prime}\left(\mathbb{R}^{3}\right)$ by means of

$$
C_{0}^{\infty}\left(\mathbb{R}^{3}\right)^{3} \ni h \longmapsto\left\langle F_{\varepsilon}(t), h\right\rangle=\left\langle\widetilde{u}^{\varepsilon}(t), \nabla \eta_{\varepsilon} \times T h\right\rangle .
$$

We deduce from (4) and (5) that

$$
\begin{aligned}
\left|\left\langle F_{\varepsilon}(t), h\right\rangle\right| & \leq\left\|u^{\varepsilon}(t)\right\|_{L^{2}\left(\Pi_{\varepsilon}\right)}\left\|\nabla \eta_{\varepsilon}\right\|_{L^{2}\left(\mathbb{R}^{3}\right)}\|T h\|_{L^{\infty}(B(0,2 \varepsilon M))} \\
& \leq C \varepsilon^{\frac{3}{2}}\left\|u_{0}^{\varepsilon}\right\|_{L^{2}\left(\Pi_{\varepsilon}\right)}\|h\|_{H^{2}\left(\mathbb{R}^{3}\right)} .
\end{aligned}
$$

Remembering that $u^{\varepsilon} \in C_{w}^{0}\left([0, \infty) ; H_{\varepsilon}\right)$ we infer that $F_{\varepsilon}$ belongs to $C_{w}^{0}([0, \infty)$; $\left.H^{-2}\left(\mathbb{R}^{3}\right)\right)$ and is bounded by $C \varepsilon^{\frac{3}{2}}$ in $L^{\infty}\left(\mathbb{R}_{+} ; H^{-2}\left(\mathbb{R}^{3}\right)\right)$. From (8) one has that

$$
\left|\left\langle\eta_{\varepsilon} \widetilde{u}^{\varepsilon}(t)+F_{\varepsilon}(t)-\eta_{\varepsilon} \widetilde{u}^{\varepsilon}(s)-F_{\varepsilon}(s), \phi\right\rangle\right| \leq C(t-s)^{\frac{1}{2}}\|\phi\|_{H^{3}\left(\mathbb{R}^{3}\right)},
$$

so

$$
\left\|\mathbb{P}\left[\eta_{\varepsilon} \widetilde{u}^{\varepsilon}(t)+F_{\varepsilon}(t)-\eta_{\varepsilon} \widetilde{u}^{\varepsilon}(s)-F_{\varepsilon}(s)\right]\right\|_{H^{-3}\left(\mathbb{R}^{3}\right)} \leq C(t-s)^{\frac{1}{2}},
$$

where $\mathbb{P}$ denotes the usual Leray projector in $\mathbb{R}^{3}$, i.e. the $L^{2}$ orthogonal projection on the subspace of divergence-free vector fields. We conclude that the set $\mathbb{P}\left(\eta_{\varepsilon} \widetilde{u}^{\varepsilon}+F_{\varepsilon}\right)$ is equicontinuous (in time) in $C^{0}\left([0, \infty) ; H^{-3}\left(\mathbb{R}^{3}\right)\right)$.

Next, we observe that $\operatorname{div} \widetilde{u}^{\varepsilon}=0$, so $\mathbb{P}\left(\widetilde{u}^{\varepsilon}\right)=\widetilde{u}^{\varepsilon}$. Therefore

$$
\mathbb{P}\left(\eta_{\varepsilon} \widetilde{u}^{\varepsilon}+F_{\varepsilon}\right)=\widetilde{u}^{\varepsilon}+\mathbb{P}\left[\left(\eta_{\varepsilon}-1\right) \widetilde{u}^{\varepsilon}+F_{\varepsilon}\right] \equiv \widetilde{u}^{\varepsilon}+v_{\varepsilon} .
$$

We argue now that

$$
\left\|v_{\varepsilon}\right\|_{L^{\infty}\left(\mathbb{R}_{+} ; H^{-3}\left(\mathbb{R}^{3}\right)\right)} \leq C \varepsilon^{\frac{3}{2}} .
$$

Indeed, we know that $\mathbb{P}$ is bounded in any $H^{s}$, so

$$
\left\|\mathbb{P} F_{\varepsilon}\right\|_{L^{\infty}\left(\mathbb{R}_{+} ; H^{-3}\left(\mathbb{R}^{3}\right)\right)} \leq C\left\|F_{\varepsilon}\right\|_{L^{\infty}\left(\mathbb{R}_{+} ; H^{-3}\left(\mathbb{R}^{3}\right)\right)} \leq C \varepsilon^{\frac{3}{2}}
$$

and

$$
\begin{aligned}
\left\|\mathbb{P}\left[\left(\eta_{\varepsilon}-1\right) \widetilde{u}^{\varepsilon}\right]\right\|_{H^{-3}\left(\mathbb{R}^{3}\right)} \leq\left\|\left(\eta_{\varepsilon}-1\right) \widetilde{u}^{\varepsilon}\right\|_{H^{-3}\left(\mathbb{R}^{3}\right)} \leq C\left\|\left(\eta_{\varepsilon}-1\right) \widetilde{u}^{\varepsilon}\right\|_{L^{1}\left(\mathbb{R}^{3}\right)} \\
\leq C\left\|\eta_{\varepsilon}-1\right\|_{L^{2}\left(\mathbb{R}^{3}\right)}\left\|\widetilde{u}^{\varepsilon}\right\|_{L^{2}\left(\mathbb{R}^{3}\right)} \leq C \varepsilon^{\frac{3}{2}}\left\|u_{0}^{\varepsilon}\right\|_{L^{2}\left(\mathbb{R}^{3}\right)}
\end{aligned}
$$

uniformly with respect to $t$. 
3.2. Passing to the limit. Given the bounds (44) and by the Ascoli theorem, we can extract from the sequence $\widetilde{u}^{\varepsilon}$ a subsequence $\widetilde{u}^{\varepsilon_{k}}$ such that

$$
\begin{gathered}
\widetilde{u}^{\varepsilon_{k}} \rightarrow u \quad \text { in } L^{\infty}\left(\mathbb{R}_{+} ; L^{2}\left(\mathbb{R}^{3}\right)\right) \text { weak*, } \\
\widetilde{u}^{\varepsilon_{k}} \rightarrow u \quad \text { in } L_{l o c}^{2}\left([0, \infty) ; H^{1}\left(\mathbb{R}^{3}\right)\right) \text { weakly, } \\
\mathbb{P}\left(\eta_{\varepsilon_{k}} \widetilde{u}^{\varepsilon_{k}}+F_{\varepsilon_{k}}\right)=\widetilde{u}^{\varepsilon_{k}}+v_{\varepsilon_{k}} \rightarrow w \quad \text { in } C^{0}\left([0, \infty) ; H_{l o c}^{-4}\left(\mathbb{R}^{3}\right)\right) \text { strongly }
\end{gathered}
$$

for some limit vector fields $u$ and $w$ where

$$
u \in L^{\infty}\left(\mathbb{R}_{+} ; L^{2}\left(\mathbb{R}^{3}\right)\right) \cap L_{\text {loc }}^{2}\left([0, \infty) ; H^{1}\left(\mathbb{R}^{3}\right)\right), \quad w \in C^{0}\left([0, \infty) ; H_{\text {loc }}^{-4}\left(\mathbb{R}^{3}\right)\right) .
$$

Since $\operatorname{div} \widetilde{u}^{\varepsilon_{k}}=0$ we necessarily have that $\operatorname{div} u=0$. Next, from (9) and (12) we infer that

$$
\widetilde{u}^{\varepsilon_{k}} \rightarrow w \quad \text { in } L_{l o c}^{\infty}\left([0, \infty) ; H_{l o c}^{-4}\left(\mathbb{R}^{3}\right)\right) \text { strongly. }
$$

Next, using that $\widetilde{u}^{\varepsilon_{k}}$ is bounded in $L_{l o c}^{2}\left([0, \infty) ; H^{1}\left(\mathbb{R}^{3}\right)\right)$ and that the interpolation inequality $\|\cdot\|_{L^{2}(W)} \leq C\|\cdot\|_{H^{-4}(W)}^{\frac{1}{5}}\|\cdot\|_{H^{1}(W)}^{\frac{4}{5}}$ holds true for every bounded open set $W$, we conclude that $\widetilde{u}^{\varepsilon_{k}} \rightarrow w$ strongly in $L_{\text {loc }}^{2}\left(\mathbb{R}_{+} \times \mathbb{R}^{3}\right)$. By uniqueness of limits in the sense of distributions, we infer that $u=w$ and therefore

$$
\widetilde{u}^{\varepsilon_{k}} \rightarrow u \quad \text { in } L_{l o c}^{2}\left(\mathbb{R}_{+} \times \mathbb{R}^{3}\right) \text { strongly. }
$$

With these pieces of information, it is easy to pass to the limit in the equation of $u^{\varepsilon_{k}}$ and obtain that $u$ is a weak solution of the Navier-Stokes equations in $\mathbb{R}^{3}$. Indeed, let $\varphi \in C_{0}^{\infty}\left([0, \infty) \times \mathbb{R}^{3}\right)$ be a divergence-free test vector field and define $\varphi_{\varepsilon_{k}}$ as in Section 2. Equation (3) with $\varphi_{\varepsilon_{k}}$ instead of $\varphi$ gives

$-\int_{0}^{\infty} \int_{\mathbb{R}^{3}} \widetilde{u}^{\varepsilon_{k}} \cdot \partial_{t} \varphi_{\varepsilon_{k}}+\nu \int_{0}^{\infty} \int_{\mathbb{R}^{3}} \nabla \widetilde{u}^{\varepsilon_{k}} \cdot \nabla \varphi_{\varepsilon_{k}}-\int_{0}^{\infty} \int_{\mathbb{R}^{3}}\left(\widetilde{u}^{\varepsilon_{k}} \otimes \widetilde{u}^{\varepsilon_{k}}\right) \cdot \nabla \varphi_{\varepsilon_{k}}=\int_{\mathbb{R}^{3}} \widetilde{u}_{0}^{\varepsilon_{k}} \cdot \varphi_{\varepsilon_{k}}(0)$.

From Lemma 4 applied to $\partial_{t} \varphi$ (see also Remark [5) we know that $\partial_{t} \varphi_{\varepsilon_{k}} \rightarrow \partial_{t} \varphi$ strongly in $L^{1}\left(\mathbb{R}_{+} ; L^{2}\left(\mathbb{R}^{3}\right)\right)$, that $\varphi_{\varepsilon_{k}}(0) \rightarrow \varphi(0)$ strongly in $L^{2}\left(\mathbb{R}^{3}\right)$ and that $\nabla \varphi_{\varepsilon_{k}} \rightarrow \nabla \varphi$ strongly in $L^{2}\left(\mathbb{R}_{+} \times \mathbb{R}^{3}\right)$. Given (10), (11) and the convergence of the initial velocity (note that only weak convergence in $L^{2}$ is required at this point for $\widetilde{u}_{0}^{\varepsilon_{k}}$ ), we deduce that the right-hand side and the first two terms on the left-hand side of (14) converge to the expected limit. Using the decomposition

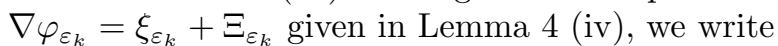

$$
\int_{0}^{\infty} \int_{\mathbb{R}^{3}}\left(\widetilde{u}^{\varepsilon_{k}} \otimes \widetilde{u}^{\varepsilon_{k}}\right) \cdot \nabla \varphi_{\varepsilon_{k}}=\int_{0}^{\infty} \int_{\mathbb{R}^{3}}\left(\widetilde{u}^{\varepsilon_{k}} \otimes \widetilde{u}^{\varepsilon_{k}}\right) \cdot \xi_{\varepsilon_{k}}+\int_{0}^{\infty} \int_{\mathbb{R}^{3}}\left(\widetilde{u}^{\varepsilon_{k}} \otimes \widetilde{u}^{\varepsilon_{k}}\right) \cdot \Xi_{\varepsilon_{k}} .
$$

Given (13) and that $\xi_{\varepsilon_{k}} \rightarrow \nabla \varphi$ weak* in $L^{\infty}\left(\mathbb{R}_{+} \times \mathbb{R}^{3}\right)$ with supports contained in a compact set independent of $\varepsilon_{k}$, one has that

$$
\int_{0}^{\infty} \int_{\mathbb{R}^{3}}\left(\widetilde{u}^{\varepsilon_{k}} \otimes \widetilde{u}^{\varepsilon_{k}}\right) \cdot \xi_{\varepsilon_{k}} \stackrel{\varepsilon_{k} \rightarrow 0}{\longrightarrow} \int_{0}^{\infty} \int_{\mathbb{R}^{3}}(u \otimes u) \cdot \nabla \varphi .
$$

Next, we use the Sobolev embedding $H^{1} \hookrightarrow L^{6}$ and a Hölder inequality to write

$$
\begin{aligned}
\left|\int_{0}^{\infty} \int_{\mathbb{R}^{3}}\left(\widetilde{u}^{\varepsilon_{k}} \otimes \widetilde{u}^{\varepsilon_{k}}\right) \cdot \Xi_{\varepsilon_{k}}\right| \leq C & \int_{0}^{\mathcal{T}}\left\|\widetilde{u}^{\varepsilon_{k}}\right\|_{L^{6}\left(\mathbb{R}^{3}\right)}^{2}\left\|\Xi_{\varepsilon_{k}}\right\|_{L^{\frac{3}{2}}\left(\mathbb{R}^{3}\right)} \\
& \leq C\left\|\widetilde{u}^{\varepsilon_{k}}\right\|_{L^{2}\left((0, \mathcal{T}) ; H^{1}\right)}^{2}\left\|\Xi_{\varepsilon_{k}}\right\|_{L^{\infty}\left((0, \mathcal{T}) ; L^{\frac{3}{2}}\right)} \stackrel{\varepsilon_{k} \rightarrow 0}{\longrightarrow} 0
\end{aligned}
$$

where $\mathcal{T}$ is such that $\operatorname{supp} \varphi \subset[0, \mathcal{T}] \times \mathbb{R}^{3}$. 
We conclude that sending $\varepsilon_{k} \rightarrow 0$ in (14) results in

$$
-\int_{0}^{\infty} \int_{\mathbb{R}^{3}} u \cdot \partial_{t} \varphi+\nu \int_{0}^{\infty} \int_{\mathbb{R}^{3}} \nabla u \cdot \nabla \varphi-\int_{0}^{\infty} \int_{\mathbb{R}^{3}}(u \otimes u) \cdot \nabla \varphi=\int_{\mathbb{R}^{3}} u_{0} \cdot \varphi(0),
$$

which is the weak formulation of the Navier-Stokes equations in $\mathbb{R}^{3}$.

To finish the proof of Theorem 7 it remains to prove that the solution $u$ is weakly continuous in time with values in $L^{2}$ and that it satisfies the energy inequality.

We show first that $u(t) \in L^{2}$ for all $t \geq 0$. Recall that $u \in C^{0}\left([0, \infty) ; H_{l o c}^{-4}\right)$ so that $u(t)$ is defined for all $t \geq 0$. For fixed $t$, by the energy inequality (4) the sequence $\widetilde{u}^{\varepsilon_{k}}(t)$ is bounded in $L^{2}\left(\mathbb{R}^{3}\right)$. Moreover, $\widetilde{u}^{\varepsilon_{k}}(t) \rightarrow u(t)$ in $H_{l o c}^{-4}$, so the limit $u(t)$ must belong to $L^{2}\left(\mathbb{R}^{3}\right)$.

Next, using again that $\widetilde{u}^{\varepsilon_{k}}(t) \rightarrow u(t)$ in $H_{l o c}^{-4}$ we have that $\int\left[\widetilde{u}^{\varepsilon_{k}}(t)-u(t)\right] \cdot h \rightarrow 0$ for all $h \in C_{0}^{\infty}\left(\mathbb{R}^{3}\right)^{3}$. On the other hand, $C_{0}^{\infty}\left(\mathbb{R}^{3}\right)$ is dense in $L^{2}\left(\mathbb{R}^{3}\right)$ and $\widetilde{u}^{\varepsilon_{k}}(t)$ is bounded in $L^{2}$, so $\int\left[\widetilde{u}^{\varepsilon_{k}}(t)-u(t)\right] \cdot h \rightarrow 0$ for all $h \in L^{2}\left(\mathbb{R}^{3}\right)^{3}$. Therefore, for all $t \geq 0$ one has that $\widetilde{u}^{\varepsilon_{k}}(t) \rightarrow u(t)$ in $L^{2}$ weakly. One can prove in a similar manner that $\nabla \widetilde{u}^{\varepsilon_{k}} \rightarrow \nabla u$ weakly in $L^{2}\left((0, t) \times \mathbb{R}^{3}\right)$ for all $t \geq 0$ and also that $u$ is weakly continuous in time with values in $L^{2}$.

We prove now that the energy inequality holds true for $u$. This is done by means of the following classical liminf argument. We apply the $\liminf _{\varepsilon_{k} \rightarrow 0}$ to (4) to obtain

$$
\liminf _{\varepsilon_{k} \rightarrow 0}\left\|\widetilde{u}^{\varepsilon_{k}}(t)\right\|_{L^{2}\left(\mathbb{R}^{3}\right)}^{2}+2 \nu \liminf _{\varepsilon_{k} \rightarrow 0} \int_{0}^{t}\left\|\nabla \widetilde{u}^{\varepsilon_{k}}\right\|_{L^{2}\left(\mathbb{R}^{3}\right)}^{2} \leq\left\|u_{0}\right\|_{L^{2}\left(\mathbb{R}^{3}\right)}^{2} \quad \forall t \geq 0 .
$$

Since $\widetilde{u}^{\varepsilon_{k}}(t) \rightarrow u(t)$ in $L^{2}$ weakly and $\nabla \widetilde{u}^{\varepsilon_{k}} \rightarrow \nabla u$ weakly in $L^{2}\left((0, t) \times \mathbb{R}^{3}\right)$ we have that

$$
\|u(t)\|_{L^{2}\left(\mathbb{R}^{3}\right)} \leq \liminf _{\varepsilon_{k} \rightarrow 0}\left\|\widetilde{u}^{\varepsilon_{k}}(t)\right\|_{L^{2}\left(\mathbb{R}^{3}\right)}
$$

and

$$
\|\nabla u(t)\|_{L^{2}\left((0, t) \times \mathbb{R}^{3}\right)} \leq \liminf _{\varepsilon_{k} \rightarrow 0}\left\|\nabla \widetilde{u}^{\varepsilon_{k}}(t)\right\|_{L^{2}\left((0, t) \times \mathbb{R}^{3}\right)} .
$$

The energy inequality for $u$ now follows from Equations (15), (16) and (17). The proof of Theorem 7 is completed.

Remark 8. It is clear from the proof that if we assume that the initial velocities $u_{0}^{\varepsilon_{k}}$ converge only weakly to $u_{0}$, then we can still prove convergence of $u^{\varepsilon_{k}}$ to some solution $u$ of the Navier-Stokes equation in the sense of Definition 2 but without the energy inequality. The strong convergence of $u_{0}^{\varepsilon_{k}}$ to $u_{0}$ is required only to prove the energy inequality.

\section{LOWER REGULARITY OF THE INITIAL VELOCITY}

The main issue in this work is to prove convergence of the solutions, and not to consider the weakest possible regularity of the initial vorticity. As we are dealing with weak solutions, however, it is natural to ask that the initial vorticity have only enough regularity to obtain existence of the weak solutions while still obtaining convergence. We give next just an example of how one can improve these regularity assumptions if the support of $\omega_{0}$ excludes the origin.

Proposition 9. Let $\omega_{0}$ be a divergence-free vector field in $L^{1}\left(\mathbb{R}^{3}\right) \cap \dot{H}^{-1}\left(\mathbb{R}^{3}\right)$ with compact support contained in $\mathbb{R}^{3} \backslash\{0\}$ and let $u_{0}$ be the unique square-integrable divergence-free vector field in $\mathbb{R}^{3}$ whose curl is $\omega_{0}$. For sufficiently small $\varepsilon$ there 
exists exactly one vector field $u_{0}^{\varepsilon} \in H_{\varepsilon}$ such that $\operatorname{curl} u_{0}^{\varepsilon}=\omega_{0}$ and there exists a constant $C$ independent of $\varepsilon$ such that $\left\|\widetilde{u}_{0}^{\varepsilon}-u_{0}\right\|_{L^{2}\left(\mathbb{R}^{3}\right)} \leq C \varepsilon^{\frac{3}{2}}$.

Proof. The operator that associates to every divergence-free $\omega_{0}$ the unique divergence-free vector field $u_{0}$ of curl $\omega_{0}$ is the Fourier multiplier $-\nabla \times \Delta^{-1}$. This operator clearly sends $\dot{H}^{-1}\left(\mathbb{R}^{3}\right)$ into $L^{2}\left(\mathbb{R}^{3}\right)$. Therefore, for every divergence-free $\omega_{0} \in \dot{H}^{-1}\left(\mathbb{R}^{3}\right)$ there exists a unique divergence-free $u_{0} \in L^{2}\left(\mathbb{R}^{3}\right)$ such that curl $u_{0}=$ $\omega_{0}$. Moreover, $\left\|u_{0}\right\|_{L^{2}} \leq C\left\|\omega_{0}\right\|_{\dot{H}^{-1}}$.

Next, let $\varepsilon$ be sufficiently small such that $\operatorname{supp} \omega_{0} \subset \Pi_{\varepsilon}$. We observe that the existence and uniqueness of $u_{0}^{\varepsilon}$ follows exactly as in the proof of Proposition 6 . Indeed, in that part of the proof the only assumption that was used is that $u_{0} \in L^{2}$.

We prove now the bound on $\left\|\widetilde{u}_{0}^{\varepsilon}-u_{0}\right\|_{L^{2}\left(\mathbb{R}^{3}\right)}$. We consider first the case when $\omega_{0}$ is smooth. More precisely, we show that for any $\delta>0$ there exists $\varepsilon_{0}=\varepsilon_{0}(\delta, M)$ and $K=K(\delta, M)$ such that for any divergence-free $\omega_{0} \in C_{0}^{\infty}\left(\mathbb{R}^{3} \backslash B(0, \delta)\right)$ and $\varepsilon \leq \varepsilon_{0}$, one has that $\left\|\widetilde{u}_{0}^{\varepsilon}-u_{0}\right\|_{L^{2}\left(\mathbb{R}^{3}\right)} \leq K \varepsilon^{\frac{3}{2}}\left\|\omega_{0}\right\|_{L^{1}}$.

In this case, relation (11) holds true and the proof is the same as that of Proposition [6 except that instead of $\psi=T u_{0}$ we use $\psi=E * \omega_{0}-E * \omega_{0}(0)$, where $E=\frac{1}{4 \pi|x|}$. This is a valid replacement because relation (11) immediately implies that $\operatorname{curl} \psi=u_{0}$. In fact, this definition of $\psi$ is equivalent to that of Proposition 6 but we don't need to prove this. Because the support of $\omega_{0}$ excludes $B(0, \delta)$, $\nabla \psi=\left(\chi_{B(0, \delta / 2)^{c}} \nabla E\right) * \omega_{0}$ on $B(0,2 M \varepsilon)$ for all $\varepsilon<\delta /(4 M)$. Here, $\chi_{B(0, \delta / 2)^{c}}$ denotes the characteristic function of $\mathbb{R}^{3} \backslash B(0, \delta / 2)$. But then

$$
\begin{aligned}
\|\nabla \psi\|_{L^{\infty}(B(0,2 M \varepsilon))} & \leq\left\|\left(\chi_{B(0, \delta / 2)^{c}} \nabla E\right) * \omega_{0}\right\|_{L^{\infty}\left(\mathbb{R}^{3}\right)} \\
& \leq\left\|\chi_{B(0, \delta / 2)^{c}} \nabla E\right\|_{L^{\infty}}\left\|\omega_{0}\right\|_{L^{1}}=\frac{1}{\pi \delta^{2}}\left\|\omega_{0}\right\|_{L^{1}} .
\end{aligned}
$$

Similarly, under the same condition $\varepsilon<\delta /(4 M)$ one can use (1) to bound

$$
\left\|u_{0}\right\|_{L^{\infty}(B(0,2 M \varepsilon))}=\left\|\int_{|x-y|>\delta / 2} \frac{x-y}{4 \pi|x-y|^{3}} \times \omega_{0}(y) d y\right\|_{L^{\infty}(B(0,2 M \varepsilon))} \leq \frac{1}{\pi \delta^{2}}\left\|\omega_{0}\right\|_{L^{1}} .
$$

With these bounds, Equation (7) can be rewritten as follows:

$$
\begin{aligned}
\left\|\widetilde{u}_{0}^{\varepsilon}-u_{0}\right\|_{L^{2}\left(\mathbb{R}^{3}\right)} & \leq\left\|\nabla \eta_{\varepsilon}\right\|_{L^{2}}\|\psi\|_{L^{\infty}(B(0,2 M \varepsilon))}+2\left\|u_{0}\right\|_{L^{2}(B(0,2 M \varepsilon))} \\
& \leq C \varepsilon^{3 / 2}\|\nabla \psi\|_{L^{\infty}(B(0,2 M \varepsilon))}+C \varepsilon^{3 / 2}\left\|u_{0}\right\|_{L^{\infty}(B(0,2 M \varepsilon))} \\
& \leq C \frac{\varepsilon^{3 / 2}}{\delta^{2}}\left\|\omega_{0}\right\|_{L^{1}} .
\end{aligned}
$$

This completes the proof when $\omega_{0}$ is smooth. The general case classically follows by approximation. Let $\omega_{0} \in L^{1} \cap \dot{H}^{-1}$ and $\delta$ be such that $\operatorname{supp} \omega_{0} \cap B(0,2 \delta)=\emptyset$. Let $\rho$ be a standard mollifying kernel and let us mollify $\omega_{0}$ in a classical manner: $\omega_{0, n}=\rho_{1 / n} * \omega_{0}$. One has that $\omega_{0, n} \rightarrow \omega_{0}$ in $L^{1} \cap \dot{H}^{-1}$ and $\omega_{0, n} \in C_{0}^{\infty}\left(\mathbb{R}^{3} \backslash B(0, \delta)\right)$ for $n$ sufficiently large. Denoting by $u_{0, n}$ and $u_{0, n}^{\varepsilon}$ the velocities associated to the vorticity $\omega_{0, n}$, we observe that $u_{0, n}=\rho_{1 / n} * u_{0}$, so $u_{0, n} \rightarrow u_{0}$ in $L^{2}$. Suppose that $\varepsilon \leq \min \left(\varepsilon_{0}, \delta\right)$. Applying the previous part of the proof to $\omega_{0, n}-\omega_{0, m}$ we get that

$$
\begin{aligned}
\| u_{0, n}^{\varepsilon} & -u_{0, m}^{\varepsilon}\left\|_{L^{2}\left(\Pi_{\varepsilon}\right)} \leq\right\| \widetilde{u}_{0, n}^{\varepsilon}-u_{0, n}-\widetilde{u}_{0, m}^{\varepsilon}+u_{0, m}\left\|_{L^{2}\left(\mathbb{R}^{3}\right)}+\right\| u_{0, n}-u_{0, m} \|_{L^{2}\left(\mathbb{R}^{3}\right)} \\
& \leq K \varepsilon^{\frac{3}{2}}\left\|\omega_{0, n}-\omega_{0, m}\right\|_{L^{1}\left(\mathbb{R}^{3}\right)}+\left\|u_{0, n}-u_{0, m}\right\|_{L^{2}\left(\mathbb{R}^{3}\right)} \rightarrow 0 \quad \text { as } m, n \rightarrow \infty .
\end{aligned}
$$

Therefore, $u_{0, n}^{\varepsilon}$ is a Cauchy sequence in $H_{\varepsilon}$, so it converges in $H_{\varepsilon}$. Moreover, curl $\lim _{n \rightarrow \infty} u_{0, n}^{\varepsilon}=\lim _{n \rightarrow \infty} \operatorname{curl} u_{0, n}^{\varepsilon}=\lim _{n \rightarrow \infty} \omega_{0, n}=\omega_{0}$ in the sense of distributions. By 
uniqueness of $u_{\varepsilon}^{0}$, we conclude that $u_{0, n}^{\varepsilon} \rightarrow u_{0}^{\varepsilon}$ in $H_{\varepsilon}$. We apply now the previous part of the proof to $\omega_{0, n}$ to deduce that $\left\|\widetilde{u}_{0, n}^{\varepsilon}-u_{0, n}\right\|_{L^{2}\left(\mathbb{R}^{3}\right)} \leq K \varepsilon^{\frac{3}{2}}\left\|\omega_{0, n}\right\|_{L^{1}}$. Letting $n \rightarrow \infty$ we finally deduce that $\left\|\widetilde{u}_{0}^{\varepsilon}-u_{0}\right\|_{L^{2}\left(\mathbb{R}^{3}\right)} \leq K \varepsilon^{\frac{3}{2}}\left\|\omega_{0}\right\|_{L^{1}}$.

Using Proposition 9 in place of Proposition 6 gives the convergence in Theorem 1 for initial vorticity in $L^{1}\left(\mathbb{R}^{3}\right) \cap \dot{H}^{-1}\left(\mathbb{R}^{3}\right)$ compactly supported in $\mathbb{R}^{3} \backslash\{0\}$.

\section{ACKNOWLEDGMENTS}

The first author would like to thank Lorenzo Brandolese, Grzegorz Karch, Milton Lopes Filho and Helena Nussenzveig Lopes for several interesting discussions on the subject of this paper. Part of this work was done during the Special Semester in Fluid Mechanics at the Centre Interfacultaire Bernoulli, EPFL; he also wishes to express his gratitude for the hospitality received.

\section{REFERENCES}

[1] G. P. Galdi, An introduction to the mathematical theory of the Navier-Stokes equations. Vol. I, volume 38 of Springer Tracts in Natural Philosophy. Springer-Verlag, New York, 1994.

[2] E. Hopf, Über die Anfangswertaufgabe für die hydrodynamischen Grundgleichungen. Math. Nachr. 4 (1951), 213-231. MR0050423 (14:327b)

[3] D. Iftimie, M. C. Lopes Filho, and H. J. Nussenzveig Lopes, Two dimensional incompressible ideal flow around a small obstacle, Comm. Part. Diff. Eqns. 28 (2003), no. 1-2, 349-379. MR1974460 (2004d:76009)

[4] - Two-dimensional incompressible viscous flow around a small obstacle, Math. Ann. 336 (2006), no. 2, 449-489. MR2244381 (2007d:76050)

[5] M. C. Lopes Filho, Vortex dynamics in a two-dimensional domain with holes and the small obstacle limit, SIAM J. Math. Anal. 39 (2007), no. 2, 422-436. MR2338413

Université de Lyon, Université Lyon 1, CNRS, UMR 5208 Institut Camille Jordan, Bâtiment du Doyen Jean Braconnier, 43, Blvd. du 11 Novembre 1918, F-69622 Villeurbanne Cedex, France

E-mail address: dragos.iftimie@univ-lyon1.fr

Department of Mathematics, Brown University, Box 1917, Providence, Rhode Island 02912

Current address: Department of Mathematics, University of California, Riverside, 900 University Avenue, Riverside, California 92521

E-mail address: kelliher@math.ucr.edu 